EESTI NSV TEADUSTE AKADEEMIA TOIMETISED. X KOIDE

FOOSIKALIS-MATEMAATILISTE JA TEHNILISTE TEADUSTE SEERIA, 1961, NR. 1

ИЗВЕСТИЯ АКАДЕМИИ НАУК ЭСТОНСКОЙ ССР. ТОМ Х СЕРИЯ ФИЗИКО-МАТЕМАТИЧЕСКИХ И ТЕХНИЧЕСКИХ НАУК. 1961, № 1

\title{
О ВОЗМОЖНОСТИ РАЗЛИЧЕНИЯ СЛАНЦА ОТ ИЗВЕСТНЯКА ПРИ ПОМОЩИ ДВУХ ПУЧКОВ РАДИОАКТИВНОГО ИЗЛУЧЕНИЯ
}

\author{
и. БРАУН
}

Обогащение горючих ископаемых является в настоящее время одной из важнейших проблем горного дела, требующей комплексного разрешения. Наряду с другими разнообразными методами обогащения горючих ископаемых предпринимались попытки разработать и новые методы, основанные на применении измерительных устройств с использованием радиоактивных излучений. Однако до сих пор эти методы еще не получили широкого практического применения, так как с их помощью можно производить обогащение только в некоторых узких интервалах размеров обогащаемых кусков.

В настоящей работе делается попытка усовершенствовать метод контроля и обогащения, основанный на использовании радиоактивных изотопов в новой принципиальной схеме, позволяющей увеличить диапазон размеров различаемых кусков по сравнению с методом обыкновенного просвечивания ['].

\section{Комбинированный метод}

Идея комбинированного метода заключается в том, что при нем один и тот же объект одновременно просвечивается двумя излучениями, имеющими разные коэффициенты поглощения в данном веществе. После прохождения излучениями контролируемого вещества изменяется разность их интенсивностей, что позволяет оценить в некоторых интервалах размеров химический состав проверяемых веществ.

Допустим, что интенсивности этих двух пучков излучений изменяются в данном веществе по закону экспоненты. Для того, чтобы в случае отсутствня в пучке излучений кусков контролируемого материала разность их интенсивностей была равна нулю, полагаем, что начальные интенсивности их равны $Q_{0}$. Будем изучать разности интенсивностей этих излучений после прохождения одного и того же слоя вещества. Математически это выражается уравнением (1):

$$
\Delta Q=Q_{0}\left(e^{-\mu x}-e^{-\delta x}\right),
$$

где $Q_{0}$ - интенсивности обоих излучений перед поглотителем, $\Delta Q-$ разность интенсивностей после прохождения контролируемого материала, $\mu$ - линейный коэффициент ослабления гамма-излучения при энергии гамма-квантов $E_{1}, \delta$ - линейный коэффициент ослабления гамма-излучения при энергии гамма-квантов $E_{2}, x-$ толщина поглощающего слоя.

3 ENSV TA Toimetised T-1 61 
Функция $\Delta Q$ может иметь как положительные, так и отрицательные значения в зависимости от величин постоянных $\mu$ и $\delta$. При $\mu=\delta \Delta Q$ равна-нулю (фиг. 1).

Из фиг. 1 видно, что функция $\Delta Q$ имеет одно максимальное значение $\Delta Q_{\max }$ при некотором расстоянии $x_{\max }$, затем при увеличении расстояния она уменьшается и при бесконечно больших значениях $x$ приближается к нулю.

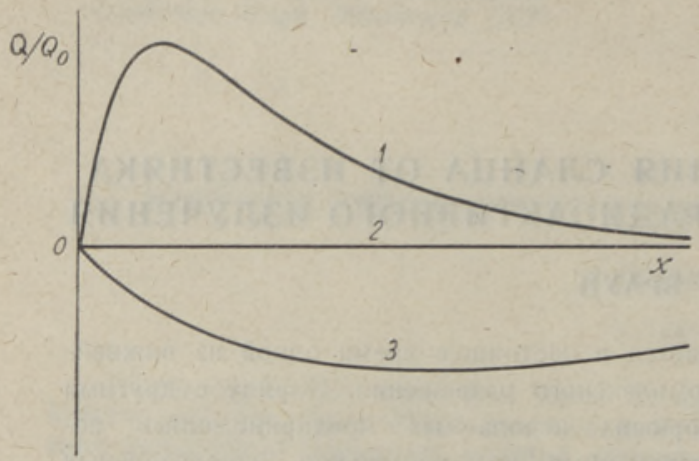

Фиг. 1. Зависимость функции $\Delta Q$ от расстояния $x$ при разных комбинациях констант $\mu$ и $\delta: 1-\mu<\delta$; $2-\mu=\delta ; 3-\mu>\delta$.

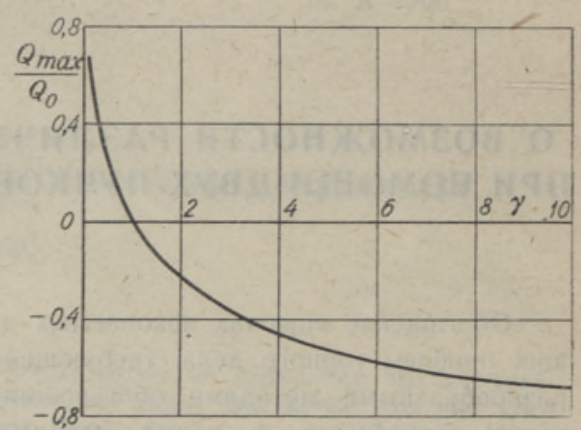

Фиг. 2. Зависимость функции $\Delta Q_{\max }$ от отношений постоянцных ослабления излучения $\frac{\delta}{\mu}=\gamma$.

Значение аргумента $x_{\max }$, при котором функция $\Delta Q$ имеет максимальное значение, можно найти, учитывая, что

$$
\left.\frac{d Q}{d x}\right|_{x=x_{\max }}=0 \text {. }
$$

Тогда после некоторых преобразований уравнения (1) получаем

$$
x_{\max }=\frac{\ln \left(\frac{\delta}{\mu}\right)}{\delta-\mu} .
$$

Заменив в уравнении (1) $x$ через $x_{\max }(3)$, получим максимальное значение функции $\Delta Q$

$$
\Delta Q_{\max }=Q_{0}\left(e^{-\frac{\delta \ln \left(\frac{\delta}{\mu}\right)}{\delta-\mu}}-e^{-\frac{\mu \ln \left(\frac{\delta}{\mu}\right)}{\delta-\mu}}\right) .
$$

Большое значение при определении значения функции $\Delta Q$ имеет отношение $\frac{\delta}{\mu}$. Обозначив его через $\gamma$, получим вместо (4) $=$

$$
\Delta Q_{\max }=Q\left(e^{-\gamma \frac{\ln \gamma}{\gamma-1}}-e^{-\frac{\ln \gamma}{\gamma-1}}\right) .
$$

Графически функция $\Delta Q_{\max }=\Delta Q_{\max }(\gamma)$ изображена на фиг. 2.

Постоянные ослабления излучения для среды без источников излучения могут иметь только положительные значения. Следовательно, отношение постоянных ослабления излучений также может иметь только положительные значения, как и показано на фиг. 2. Но в зависимости от значений $\gamma$ функция $\Delta Q_{\max }$ может иметь различные как по- 
ложительные, так и отрицательные значения в диапазоне от $Q_{0}$ до - Q. Так,

$$
\begin{array}{ll}
\text { при } 0<\gamma<1 & \Delta Q_{\max } \text { имеет только положительные значения } \\
, \quad 1<\gamma<\propto & \Delta Q_{\max }, ", \quad \text { отрицательные } \\
,, \quad \gamma=1 & \Delta Q_{\max } \text { равняется нулю. }
\end{array}
$$

Если значения функции $\Delta Q$ пропорциональны амплитудам электрических импульсов, приходящих из регистрирующего прибора, то чем больше разница между постоянными ослабления излучений, тем лучше мы можем регистрировать электрические импульсы, тем меньше будет сказываться влияние внешних условий на результаты измерений.

Если нужно различить два вещества А и Б с разными химическими составами (как это бывает при обогащении сланца), то отношение постоянных ослабления излучений $\gamma$ может иметь только два определенных значения и функция $\Delta Q$ изобразится двумя кривыми: для вещества А кривой $Q_{\mathrm{A}}$ и для вещества Б - кривой $Q_{\mathrm{b}}$. В зависимости от выбора величин $\gamma$ могут иметь место следующие случаи:

a. Пусть для обоих веществ функция $\Delta Q$ имеет только положительные или только отрицательные значения. Тогда отношение постоянных ослабления излучений может иметь следующие пары значений (фиг. 3):

$$
\begin{aligned}
& \text { 1) } 0<\gamma_{\mathrm{A}}<1 \text {, } \\
& 0<\gamma_{\mathrm{B}}<1 \text {; } \\
& \text { 2) } 1<\gamma_{\mathrm{A}}<\infty \text {, } \\
& 1<\gamma_{\mathrm{B}}<\infty \text {. }
\end{aligned}
$$

Фиг. 3. Различение вещества А (кривая $Q_{A}$ ) от вещества Б (кривая $Q_{\mathrm{E}}$ ). при $\gamma_{\mathrm{A}}, \gamma_{\mathrm{b}}$, подчиняющихся требованиям:

$$
\begin{gathered}
\gamma_{\mathrm{A}}<\gamma_{\mathrm{5}} ; 0<\left(\nu_{\mathrm{A}}, \gamma_{\mathrm{B}}\right)<1 \\
\text { (случай } a) .
\end{gathered}
$$

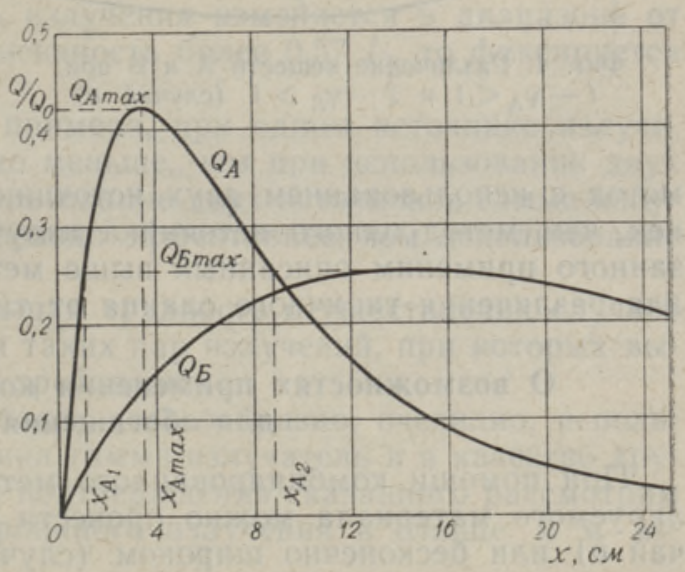

Как видно из фиг. 3, значения функуии $Q_{\mathrm{A}}$ превосходят любые значения функции $Q_{5}$ при величине аргумента $x_{\mathrm{A}_{1}}<x<x_{\mathrm{A}_{2}}$. Следовательно, по данной схеме эти вещества можно различить лишь в том случае, если размеры кусков изменяются в интервале $x_{\mathrm{A}_{1}}<x_{\mathrm{A}}<x_{\mathrm{A}_{2}}$.

Диапазон различения $x_{\wedge_{1}}-x_{A_{2}}$ расширяется, если увеличивается разница между максимальными значениями $Q_{\mathrm{A}_{\max }}$ и $Q_{\mathrm{b}_{\max }}$, которые в свою очередь зависят от постоянной $\gamma$. Следовательно, для того, чтобы увеличить диапазон размеров обогащаемых кусков, нужно выбрать излучения так, чтобы для одного вещества $\gamma$ было сравнительно большим, а для другого - сравнительно малым. При различении сланца такими излучениями могут быть, например, мягкие и жесткие гамма-излучения.

б. Легко произвести разделение кусков контролируемых веществ, если для одного материала функция $\Delta Q$ однозначно равняется нулю при всех значениях аргумента $x$, но для другого материала $\Delta Q$ может равняться нулю только при значениях $x=0$ и $x=\infty$ (при других зна- 
чениях аргумента $x$ функция $\Delta Q \neq 0$ ). В этом случае размеры анализируемых кусков могут быть любой величины.

Практически этого нелегко достигнуть, потому что трудно подобрать пару таких излучений, при которых выполнялись бы вышеупомянутые требования. Но в некотором приближении этого можно достичь, если в качестве одного излучения выбрать нейтронное излучение с соответствующим энергетическим спектром, а в качестве второго, например, гамма-излучение.

в. Очень легко различить контролируемые вещества, если для одного материала функция $\Delta Q$ имеет только отрицательные, а для другого - только положительные значения (фиг. 4). В этом случае размеры кусков могут изменяться в диапазоне от нуля до бесконечности.

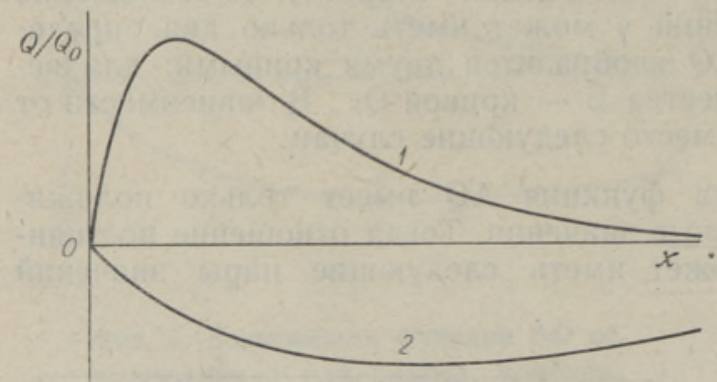

Практически этого можно достигнуть, если учитывать резонансные области поглощения нейтронного излучения в исследуемых материалах и соответственным образом комбинировать с ними энергетические спектры источников нейтронного излучения.

Для различения сланща от Фиг. 4. Различение веществ А и Б при: $1-\gamma_{\mathrm{A}}<1$ и $2-\gamma_{\mathrm{Б}}>1$ (случай $в$ ). известняка или контроля качества продуктов переработки сланца на обогатительных фабриках комбинированный метод с использованием двух источников излучения более перспективен, чем метод одного источника излучения. Для иллюстраций сказанного применим описанный выше метод-двух источников излучения для различения типичного сланца от типичного известняка.

\section{О возможностях применения комбинированного метода для обогащения сланца}

При помощи комбинированного метода различение кусков контролируемого материала можно провести в сравнительно широком (случай $a$ ) или бесконечно широком (случаи б и в) интервале размеров обогащаемых кусков [1]. Рассмотрим отдельно эти случаи различения сланца от известняка.

С лу у а й $a$. В качестве источников излучения выберем источники гамма-излучения с энергией, например, 1,3 и 0,5 Мэв. Массовый коэффициент для типичного сланца при энергии гамма-квантов $1,3 \mathrm{Mэв}$ равен 0,0769 и при энергии 0,5 Мэв $0,1178 \mathrm{~cm}^{2} / г$. Для типичного известняка эти значения равны при 1,3 Мэв 0,0635 и при 0,5 Мэв $0,1026 \mathrm{~cm}^{2} /$ г.

Сравним метод двух источников излучения с методом одного источника излучения.

Пусть на фиг. 5 представлена функция $\Delta Q$, где кривые $Q_{c}$ и $Q_{*}$ характеризуют соответственно сланец и известняк. Из этих кривых видно, что различение в данном случае можно произвести в интервале размеров обогащаемых кусков от 3,2 до 8,2 см и изменения интенсивности гамма-излучения в диапазоне от $0,165 I_{0}$ до $0,140 I_{0}$. Если интенсивность гамма-излучения больше $Q_{\mathrm{c}_{\max }}$, то в пучке излучения находится кусок известняка, если же она меньше $Q_{c_{\max }}$, то мы имеем дело со сланцем. 
При одном источнике гамма-излучения с энергией, например, 0,5 М эв различить сланец от известняка можно только в некотором очень небольшом интервале размеров обогащаемых кусков. При минималь-

Фиг. 5. Сравнение метода одного источника излучения с методом двух источников излучения при энергии излучения: для сланца $I-1,3$ Мэв, $2-0,5$ Мэв и для известняка $3-$ 1,3 Мэв, $4-0,5$ Мэв.

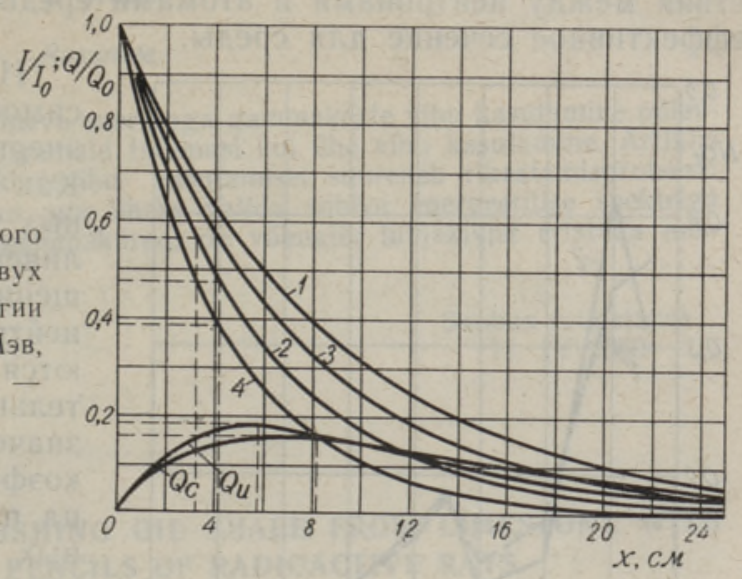

ном диаметре обогащаемых кусков $x=3,2$ см разделить можно только куски величиной от 3,2 до 4,2 см (фиг. 5). Здесь известняк будет зафиксирован, если интенсивность излучения изменяется в диапазоне от $0,47 I_{0}$ до $0,57 I_{0}$. Если же интенсивность более $0,57 I_{0}$, то фиксируется сланец.

Қак видно из приведенного примера, при одном источнике излучения диапазон различения гораздо меньше, чем при использовании двух источников. Следовательно, использование двух источников гамма-излучения при различении сланца гораздо эффективнее, чем использование только одного источника.

С луч и и б и в. Самая большая трудность осуществления случаев б и в заключается в нахождении таких пар излучений, при которых выполнялись бы упомянутые выше требования.

Для различения сланца наиболее целесообразно, очевидно, использовать в качестве одного источника гамма-излучатель и в качестве другого - источник нейтронов. Для подтверждения сказанного рассмотрим ослабление интенсивности нейтроннного излучения в сланце и в известняке.

\section{Поглощение нейтронного излучения в сланце и известняке}

Интенсивность нейтронного излучения, как и интенсивность гаммаизлучения, изменяется в среде по экспоненциальному закону [ $\left.{ }^{2}\right]$

$$
N=N_{0} e^{-n s t^{x}}
$$

где $N_{0}$ - интенсивность нейтронного излучения перед поглотителем, $N$ - интенсивность после поглощения, $\sigma_{t}-$-эффективное сечение поглощения элементов для нейтронного излучения, $n-$ число атомов в единице объема, $x$ - толщина поглощающего слоя.

Эффективное сечение поглощения нейтронного излучения элементов $\sigma_{t}$ находится в очень сложной зависимости от энергии. Если эту зависимость представить графически, то кривая будет иметь несколько минимумов и максимумов. Однако в целом абсолютные значения $\sigma_{t}$ при больших значениях энергии увеличиваются с увеличением массового числа $A\left[{ }^{3}\right]$. 
Если длины волн нейтронов (быстрые нейтроны) выбрать так, чтобы можно было пренебречь эффектами от кристаллических решеток вследствие их незначительности, то, учитывая аддитивность взаимодействия между нейтронами и атомами среды, можно вычислить среднее эффективное сечение для среды.

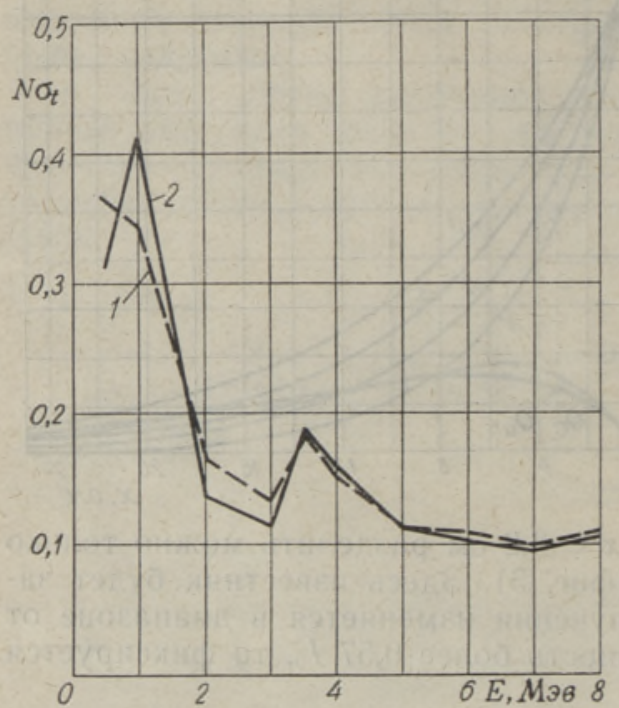

Фиг. 6. Зависимость линейных коэффициентов поглощения нейтронного излучения $N \sigma_{t}$ для сланца $(1)$ и известняка (2) от энергии $E$.

На фиг. 6 представлена зависимость эффективного сечения от энергии для сланца и известняка. Как видно из графика, при разных значениях энергии нейтронов линейные коэффициенты поглощения сланца и известняка для нейтронного излучения не остаются постоянными друг относительно друга. При некоторых значениях энергии линейные коэффициенты поглощения сланца превышают значения линейных коэффициентов поглошения известняка, при других же значениях энергии, наоборот, линейные коэффициенты поглошения известняка превышают значения коэффициентов поглощения сланца. Если выбрать источник нейтронов с соответствующим спектром, то можно добиться такого эффекта, что суммарный коэффициент поглошения обоих веществ будет почти одинаков или же достичь того, чтобы для гамма-излучения коэффициент поглощения сланца оказался больше, чем коэффициент поглощения известняка. В сущности это означает, что могут быть реализованы случаи, описанные в пунктах 6 и в настоящей работы (стр. 35 и 36 ).

\section{ЛИТЕР АТ У РА}

1. J. B r a u n, Ohest radioaktiivsete isotoopide kasutamisvõimalusest põlevkivi rikastamise automatiseerimisel, Изв. АН ЭССР. Серия физ.-мат. и техн. наук, т. IX, № 4, 1960.

2. Д. Ю з, Нейтронные исследования на ядерных котлах, ИЛ, М., 1954.

3. Атлас эффективных нейтронных сечений элементов (ред. И. В. Ад ам чук), М., 1955.

Институт кибернетики Академии наук Эстонской ССР
Поступила в редакцию 11. IV 1960 


\section{POLEVKIVI ERISTAMISE VOIMALUSEST LUBJAKIVIST KAHE RADIOAKTIIVSE KIIRGUSE VIHU ABIL}

\section{Braun}

Resümee

Nagu on selgunud, annab kahe erineva energiaga gammakiirte vihu kasutamine põlevkivi eristamiseks lubjakivist märksa paremaid tulemusi kui ühe vihu kasutamine. Artiklis esitatud näited kinnitavad, et kahe kiirtevihu kasutamisel suureneb rikastamisprotsessi efektiivsus ligikaudu viiekordseks. Kui aga üheks valida sobiva energeetilise spektriga neutronkiirte vihk ja teiseks vihuks gammakiired, on vōimalik lubjakivist eristada mis. tahes tükisuurusega pōlevkivi.

\section{Eesti NSV Teaduste Akadeemia} Küberneetika Instituut

Saabus toimetusse 11. IV 1960

\section{THE POSSIBILITY OF DISTINGUISHING OIL SHALE FROM LIMESTONE WITH THE HELP OF TWO PENCILS OF RADIOACTIVE RAYS}

\section{Braun}

\section{Summary}

It has been stated that the application of two pencils of gamma rays of different energies yields better results at the distinguishing of oil shale from limestone than the application of one pencil of rays. The examples presented in the article show that the process of enrichment, at the application of two pencils of rays, becomes about five times more effective. If, however, one pencil of neutron rays with a suitable energetic centre is taken along with a pencil of gamma rays, it becomes possible to discern pieces of shale of any size from limestone.

$$
\begin{gathered}
\text { Academy of Sciences of the Estonian S.S.R., } \\
\text { Institute of Cybernetics }
\end{gathered}
$$

Received April 11th, 1960 